\title{
Cavitation rheology of the vitreous: mechanical properties of biological tissue
}

\author{
Jessica A. Zimberlin, ${ }^{a}$ Jennifer J. McManus ${ }^{* b}$ and Alfred J. Crosby ${ }^{* a}$ \\ Received 3rd December 2009, Accepted 30th April 2010 \\ DOI: 10.1039/b925407b
}

\begin{abstract}
We demonstrate the importance of measuring the mechanical properties of biological tissue in vivo by quantifying the mechanical properties of bovine vitreous both in its native state and upon removal from the eye. The mechanical properties are determined by the cavitation rheology technique developed in our labs to determine the linear modulus. This technique involves inducing a cavitation event at the tip of a syringe that is located at an arbitrary location within the vitreous sample. The pressure at which the cavitation event occurs can be directly related to the modulus. We show that the modulus decreases upon removal of the vitreous from the ocular cavity and decreases still further when the vitreous cortex, the thin membrane that surrounds the vitreous, is removed. This study has important implications for both diagnostic science of diseased tissue and developmental biology in studying tissue properties with age.
\end{abstract}

\section{Introduction}

Measuring the mechanical properties of tissue in vivo is critical to developing knowledge of how these properties change with age and, more importantly, how they change upon the onset and through the progression of disease. ${ }^{1-3}$ Current methods for the in vivo characterization of animal tissue include two widely used techniques: palpation and elastographic imaging. Palpation is when an individual feels the tissue for regions of either increased or decreased stiffness. ${ }^{4}$ This technique is generally relied upon for the early detection of diseased areas due to its non-invasiveness, but it can only provide qualitative information since it is highly dependent on the palpator's acumen. ${ }^{4}$ To provide quantitative understanding to the differences measured with palpation, various versions of elastographic imaging techniques, such as magnetic resonance elastography and ultrasound elastography, have been developed over the past decade to image a tissue's strain response after imposition of a known stress., ${ }^{1,5-8}$ Combining the images and known stress/strain relationships, these techniques can provide "elasticity" images representing stiffness maps of the tissue. Although non-invasive and pictorial, these imaging techniques are limited as the transformation of the strains measured to local moduli is quite complex. ${ }^{9}$ Therefore, there is clearly a need to link the relative information displayed in elastographic images to quantitative in vivo measurements of elastic constants.

In general, most quantitative information is acquired from ex vivo techniques such as shear rheometry, compression or tension testing, contact mechanics, or microbead rheology. While these techniques have been instrumental in tissue characterization, they require the removal of the tissue sample from the host. This removal process not only limits the development of knowledge for tissue properties as a function of age, but it has

${ }^{a}$ Department of Polymer Science and Engineering, University of Massachusetts, 120 Governors Dr., Amherst, MA, 01003, USA. E-mail: Crosby@mail.pse.umass.edu; Fax: +1 413545 0082; Tel: +1 4135771313 ${ }^{b}$ Department of Chemistry, National University of Ireland, Maynooth, Co. Kildare, Ireland. E-mail: Jennifer.McManus@nuim.ie; Tel: +3531708 6926 also been suggested that the act of tissue removal from its native environment can significantly change its mechanical properties. ${ }^{\mathbf{1 0}}$

Recent results support this hypothesis of differences in mechanical properties between in vivo and ex vivo samples. For example, shear rheometry was performed on slices of the vitreous humor [referred to here as the vitreous] of bovine and porcine eyes as a function of time from removal from its native state. ${ }^{10}$ The results showed that the shear storage modulus of bovine vitreous decreased from $G^{\prime} \approx 32 \pm 12 \mathrm{~Pa}$ to a final steady state value of $G^{\prime} \approx 7 \pm 2 \mathrm{~Pa}$ over approximately sixty minutes. The authors attribute this modulus decrease to failure of the vitreous network by the onset of phase separation upon removal from the sclera, and postulate that the modulus of the vitreous in vivo, before any separation occurs, may be greater than the values they measured at early times.

The vitreous is a clear hydrogel network that is $99 \mathrm{wt} \%$ water, $0.9 \mathrm{wt} \%$ salts and proteins, and $0.1 \mathrm{wt} \%$ network components comprised mainly of fibrous collagen type II. ${ }^{11}$ These collagen fibrils are thin, ranging from 6-16 $\mathrm{nm}$ in diameter, and unbranched with an overall concentration of approximately $60 \mu \mathrm{g} \mathrm{mL}^{-1}$ in bovine eyes. The concentration of collagen is not uniform throughout the vitreous and its relative concentration in different areas defines the three primary regions of the vitreous: the central vitreous, the vitreous cortex, and the basal vitreous (Fig. 1a). The central vitreous comprises the bulk of the vitreous body. It is the region of lowest collagen concentration and is surrounded by the $0.1-0.3 \mathrm{~mm}$ thick vitreous membrane, otherwise known as the cortex, surrounding the central vitreous. The central vitreous eventually blends into the basal vitreous, the densest collagen region located at the anterior limit of the retina. ${ }^{12-14}$

The collagen fibrils are part of a hydrated double network with high molecular weight, polyanionic hyaluronan macromolecules. ${ }^{11}$ This network is responsible for maintaining the mechanical integrity of the hydrogel, where the collagen is hypothesized to act as the load-bearing struts and the hyaluronan serves to hydrate the system, imposing a tensile force on them and preventing the collagen fibrils from aggregating. ${ }^{10}$ These structural conclusions are based on depolymerization 


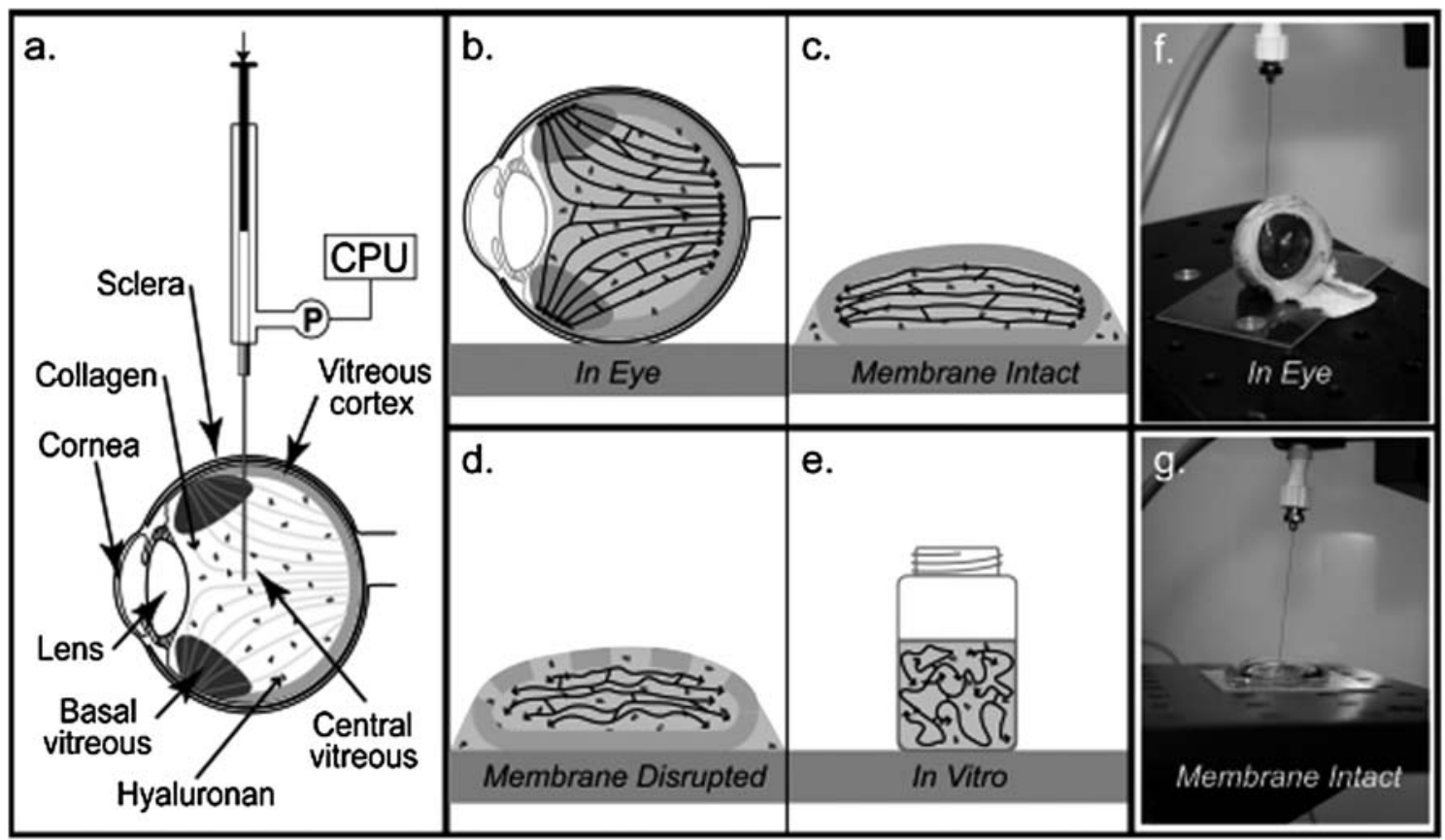

Fig. 1 Experimental set-up and vitreous map. (a) Schematic of the various regions of the eye and the cavitation rheology set-up. Schematics of the vitreous samples tested: in eye (b), membrane intact (c), membrane disrupted (d), and in vitro (e). Images of actual samples tested with the vitreous in eye (f) and with the membrane intact $(\mathrm{g})$.

experiments where digestion with collagenase completely dissolves the gel while the enzymatic removal of hyaluronan does not collapse the network. ${ }^{13,15}$ As individuals age, the collagen fibrils begin to aggregate with each other and the double hydrated network begins to fail, resulting in a slow liquefaction of the vitreous gel. Medically, this is referred to as Posterior Vitreous Detachment. It is a common condition with $65 \%$ of people over the age of 65 affected..$^{16}$ Most cases are left untreated, although a small number of cases may lead to retinal tears as the vitreous collapses from the back of the eye. In these cases surgical intervention is sometimes required. With the gel liquefaction, a decrease in mechanical properties is observed. This gel liquefaction is the primary cause of posterior vitreous detachment, ${ }^{11,14}$ and it is suggested that the same phenomenon leads to the decreased mechanical properties observed upon removal of the vitreous from its native state. ${ }^{10}$ This is also important since the vitreous may be used as a drug reservoir for pharmaceuticals in the treatment of retinal conditions. ${ }^{17,18}$ Intravitreal injection of drugs is the most common method for treatment of endophthalmitis and CMV retinitis. ${ }^{17}$ Any changes in the physical properties of the vitreous, or in any material used to replace the vitreous after surgical intervention, will possibly alter drug distribution within the eye or indeed the effectiveness of controlled release from polymeric drug delivery systems in the vitreous. ${ }^{18}$

Therefore, a measurement technique that has the capability to determine the mechanical properties of a tissue in vivo at any arbitrary location would offer invaluable insight to these differences between ex vivo and in vivo properties and their relation to the tissue's hierarchical structure.

In this paper, measurements of the elastic properties of the vitreous both in the eye and upon removal are performed by cavitation rheology [CR], which has a distinct advantage of being capable of performing in vivo characterization of mechanical properties. The technique involves inserting a syringe needle into a sample and inducing an elastic instability via slow pressurization. The pressure at which the instability occurs can be related to the local mechanical properties of the sample. For a cavitation event, the critical pressure for cavitation, $P_{\mathrm{c}}$, is: ${ }^{19-22}$

$$
P_{\mathrm{c}}=\frac{5}{6} E+\frac{2 \gamma}{r}
$$

where $E$ is the elastic modulus at low strains, $\gamma$ is the surface tension and $r$ is the radius of the needle used for the measurement. Using this equation and plotting the critical pressure for various values of $r$ provide information about both the elastic properties of the material and its surface properties. When the experiment is performed with air as the cavitation media, the cavitation event results in a small millimetre diameter sized cavity that forms at the tip of the syringe needle and rises to the surface of the sample. Although visual inspection cannot conclusively prove that no damage occurs to the soft tissue, the linear relationship between $P_{\mathrm{c}}$ and $1 / r$ is consistent with complementary results on synthetic hydrogels that demonstrated the linear scaling indicates reversible, elastic cavitation. ${ }^{19}$ Fracture, or irreversible deformation, was demonstrated to scale with $1 / \sqrt{ } r$. It should be noted that these scaling relationships are predicted for the initiation pressure, and do not describe subsequent events associated with bubble propagation or growth. Here, we use CR to quantify the mechanical properties of the vitreous in situ and the changes associated with removing this material from its native state. This characterization demonstrates the importance of measuring the mechanical properties of biological tissues in their native environment and opens new opportunities for similar future investigations on other soft tissue. 


\section{Materials and methods}

Bovine eyes from young calves were obtained from Research 87 (Boylston, Massacusetts), and used within $48 \mathrm{~h}$ post-mortem. The animals were either Jersey or Holstein males between 2 and 10 days old and were certified disease free. Eyes were obtained for two rounds of experiments, once in August 2008 and once in January 2009. We quantify the mechanical properties of the vitreous both within the sclera [in eye] (Fig. 1a, b and f) and upon removal from the ocular cavity. The vitreous samples that were tested after being removed from the eye were tested in three different states (1) with the membrane intact [membrane intact] (Fig. 1c and g), (2) with the membrane disrupted [membrane disrupted] (Fig. 1d), and (3) with the membrane completely removed so that the vitreous could no longer maintain its shape [in vitro] (Fig. 1e). The eyes were stored at $8{ }^{\circ} \mathrm{C}$ and allowed to warm to room temperature prior to experimentation. Unless otherwise stated, all experiments in which the vitreous was removed occurred within 5 minutes of removal from the sclera. For experiments performed in eye, the location of the syringe needle within the eye was monitored. The eye was centered under the syringe needle with the iris and lens positioned approximately $90^{\circ}$ to the motion stage. The syringe needle was then lowered $2.5 \mathrm{~cm}$ into a slit that was made in the sclera of the eye and the experiment was initiated.

To remove the vitreous from the eye, an incision was made between the cornea and the sclera, and the lens was removed. The vitreous was then carefully removed from its surrounding material. Preparation of the membrane removed samples involved cutting two slits in the vitreous membrane in the form of an $\mathrm{X}$ and peeling the membrane back. The in vitro samples were prepared by removing the vitreous from the eye, placing it in a vial, and gently stirring to break any connections the collagen fibrils had to the vitreous membrane.

\section{Results and discussion}

CR experiments were performed on the in eye and in vitro vitreous samples. The critical pressure at which cavitation occurred at several needle radii is shown in Fig. 2. Using eqn (1), both the modulus and the surface tension can be directly determined for both vitreous samples. The storage modulus for the vitreous in eye was found to be approximately $660 \mathrm{~Pa}$.

Once the vitreous had been removed from the ocular cavity, the measured modulus decreased to approximately $120 \mathrm{~Pa}$. This decrease in modulus, of almost six times the measured value in eye, is significant and is well below the variation in modulus that occurs due to differences between individual samples. These moduli values are higher than those previously reported in literature, ${ }^{23-25}$ with the exception of work of Nickerson et al., who determined the modulus of the vitreous in a conventional rheometer using a novel cleated geometry to prevent slippage. These authors concluded that significant network disruption occurs with removal of the vitreous from the eye. Our work has confirmed these findings and has extended it to include the measurement of the modulus of the vitreous inside the eye. The notable increase in modulus measured for the vitreous in eye highlights the need to measure the mechanical properties of biological tissue in situ and the usefulness of cavitation rheology

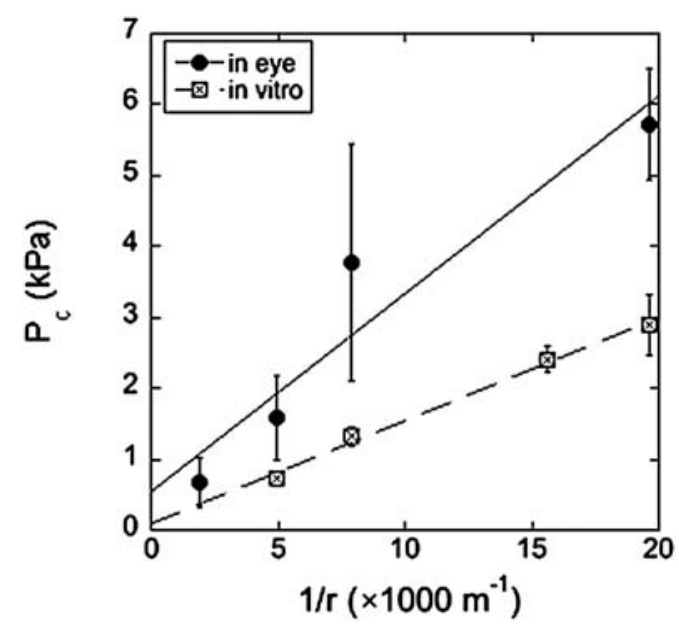

Fig. 2 The change in the critical pressure at which cavitation occurs, with decreasing needle radius for the vitreous inside the eye (in eye) and the vitreous removed from the eye (in vitro). Each data point is an average of between 5 and 10 samples.

as a technique. The surface tension values that were measured for the vitreous in eye and in vitro are approximately 0.14 and $0.07 \mathrm{~N} \mathrm{~m}^{-1}$ respectively. These values are reasonable as the vitreous is $99 \%$ water, which has a reported surface tension of $0.072 \mathrm{~N} \mathrm{~m}^{-1}$ at $25^{\circ} \mathrm{C}^{26}$

To further study the cause of the decrease in storage modulus of the vitreous upon removal from its native state, the role of the vitreous membrane was studied. This was done by probing the effect of removing the vitreous from its native state and studying the effect of liquid expulsion on the reduced tension of the collagen fibrils in the vitreous. Here, a vitreous sample was removed from the sclera with its membrane intact [a membrane intact sample] and was allowed to rest at room temperature until fluid diffused through the membrane and accumulated around the sample. The critical pressure was measured both in the vitreous gel and in the surrounding fluid and is presented in Fig. 3, left. The $P_{\mathrm{c}}$ for the vitreous has a greater mechanical contribution from the network (Fig. 3, left), but it is clear that relative to the in eye experiments there is a decrease in the average $P_{\text {c }}$ (dotted line). This implies that the fluid loss is reducing network tension on the fibrils from the native state, resulting in a decrease in modulus.

The relative importance of this membrane was then probed by comparing the instability pressure of a similar vitreous sample as the one used previously, Fig. 3, after removal from the sclera both with [membrane intact] and without the outer membrane [membrane disrupted] (Fig. 3, right). A significant decrease in $P_{\mathrm{c}}$ is measured when the membrane is removed and the vitreous assumes a more liquid-like state. This implies that the membrane is contributing to the vitreous' maintenance of its structure both in the sclera and upon removal and that it significantly contributes to the tension imposed on the collagen fibrils in vivo.

The results obtained here provide insight into the role of the sclera and the vitreous membrane on the maintenance of the mechanical properties of the vitreous. In young eyes, once the vitreous is kept within the sclera, phase separation of the vitreous fluid from the collagen fibrils does not occur. The prevention of phase separation is important for the vitreous' ability to maintain 


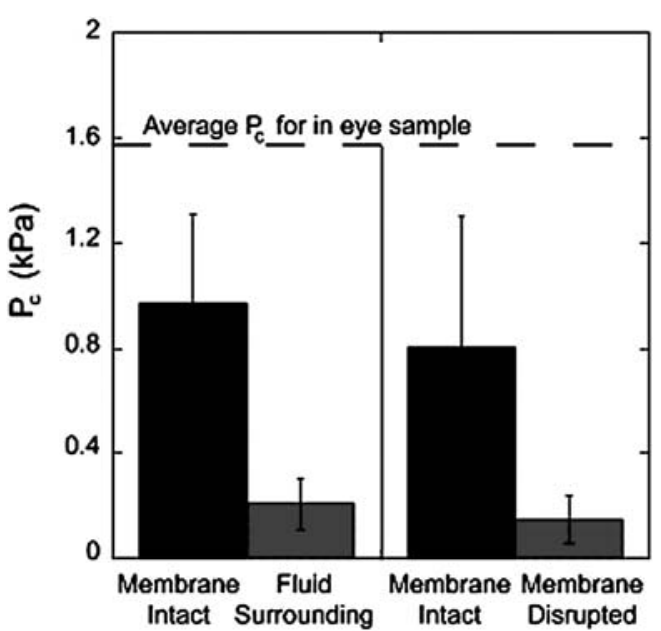

Fig. 3 Determination of the importance of the vitreous membrane where the change in critical pressure was monitored for a vitreous sample both inside the membrane and in the fluid that was expelled (left) and of a vitreous sample with its membrane intact and after its membrane was disrupted (right) $(r=203 \mu \mathrm{m})$. Each data point is an average of between 5 and 10 samples.

its mechanical integrity as fluid loss from the matrix, which includes the loss of hyaluronan, leads to a decreased tension on the collagen fibrils. This decrease in tension occurs due to the double hydrated network structure of the vitreous as described previously, where the hyaluronan imposes a tension on the collagen fibrils, preventing them from aggregating into collagenrich areas. When the vitreous is removed from the sclera, the native state is disrupted as is seen by (1) the vitreous' inability to maintain its spherical shape, (2) fluid loss from the system, and (3) by a decrease in the pressure to cavitate. Removal of the vitreous membrane further disrupts the hydrogel network as is seen in the drop in $P_{\mathrm{c}}$ on the right side plot in Fig. 3. We believe that upon disruption of the vitreous membrane, further tension on the collagen fibrils is released due to both the continued phase separation of the fluid and hyaluronan from the collagen and the disruption of the end-point attachments of the collagen fibrils to the cortex. In vivo, the collagen fibrils in the central vitreous tend to run in an anterior-posterior direction with the fibrils inserting in the anterior end into the vitreous base and into the posterior end into the vitreous membrane. As the collagen presumably acts as the load-bearing struts in the vitreous, a decrease in tension on the fibrils, due to disruption of the end-point attachment, could release an imposed tension on these fibrils thus allowing for continued network relaxation and separation.

\section{Conclusions}

In summary, we have demonstrated the use of cavitation rheology as a suitable method for determining the mechanical properties of biological tissue and have clearly shown the importance of quantifying these properties in in vivo settings. Our research has shown that the modulus of the vitreous decreases when the vitreous is removed from the eye and decreases further when the vitreous cortex is disrupted. We anticipate that the benefits of determining the mechanical properties of tissues in their native environments will open many opportunities for studying soft tissues as a function of age and disease and in developing design maps to help engineer synthetic scaffolds that more closely resemble their corresponding target tissues.

\section{Acknowledgements}

Zimberlin and Crosby would like to thank the NSF-MRSEC (NSF DMR-0820506) and McManus would like to thank Science Foundation Ireland-Stokes Lectureship for funding. McManus would also like to thank Colm O'Brien for a helpful discussion.

\section{References}

1 L. Gao, K. J. Parker, R. M. Lerner and S. F. Levinson, Ultrasound Med. Biol., 1996, 22, 959-977.

2 V. Egorov and A. P. Sarvazyan, IEEE Trans. Med. Imaging, 2008, 27, $1275-1287$.

3 J. Walraevens, B. Willaert, G. De Win, A. Ranftl, J. De Schutter and J. V. Sloten, Med. Eng. Phys., 2008, 30, 1098-1104.

4 J. F. Greenleaf, M. Fatemi and M. Insana, Annu. Rev. Biomed. Eng., $2003,5,57-78$.

5 R. Muthupillai, P. J. Rossman, D. J. Lomas, J. F. Greenleaf, S. J. Riederer and R. L. Ehman, Magn. Reson. Med., 1996, 36, 266274.

6 R. Muthupillai, D. J. Lomas, P. J. Rossman, J. F. Greenleaf, A. Manduca and R. L. Ehman, Science, 1995, 269, 1854-1857.

7 J. Ophir, I. Cespedes, H. Ponnekanti, Y. Yazdi and X. Li, Ultrason. Imaging, 1991, 13, 111-134.

8 T. A. Krouskop, T. M. Wheeler, F. Kallel, B. S. Garra and T. Hall, Ultrason. Imaging, 1998, 20, 260-274.

9 T. Varghese, E. Ophir, E. Konofagou, F. Kallel and R. Righetti, Ultrason. Imaging, 2001, 23, 216-248.

10 C. S. Nickerson, J. Park, J. A. Kornfield and H. Karageozian, J. Biomech., 2008, 41, 1840-1846.

11 P. N. Bishop, Prog. Retinal Eye Res., 2000, 19, 323-344.

12 J. Sebag, Graefes Arch. Clin. Exp. Ophthalmol., 1987, 225, 89-93.

13 K. J. Bos, D. F. Holmes, R. S. Meadows, K. E. Kadler, D. McLeod and P. N. Bishop, Micron, 2001, 32, 301-306.

14 J. Sebag, Graefes Arch. Clin. Exp. Ophthalmol., 2004, 242, 690-698.

15 J. Sebag and E. A. Balazs, Invest. Ophthalmol. Visual Sci., 1989, 30, 1867-1871.

16 F. RY, PVD, Trans. - Am. Acad. Ophthalmol. Otolaryngol., 1972, 76, 480-497.

17 S. Friedrich, Y. Cheng and B. Saville, Curr. Eye Res., 1997, 16, $663-$ 669.

18 J. Kathawate and S. Acharya, Int. J. Heat Mass Transfer, 2008, 51, $5598-5609$.

19 S. Kundu and A. J. Crosby, Soft Matter, 2009, 5, 3963-3968.

20 J. A. Zimberlin, N. Sanabria-DeLong, G. N. Tew and A. J. Crosby, Soft Matter, 2007, 3, 763-767.

21 A. N. Gent and D. A. Tompkins, J. Appl. Phys., 1969, 40, 2520-2525.

22 A. N. Gent and C. Wang, J. Mater. Sci., 1991, 26, 3392-3395.

23 R. L. Zimmerman, Biophys. J., 1980, 29, 539-544.

24 F. Bettelheim and T. Wang, Exp. Eye Res., 1976, 23, 435-441.

25 B. Lee, M. Litt and G. Buchsbaum, Biorheology, 1994, 31, 327-338.

26 C. R. Company, CRC Handbook of Chemistry and Physics, CRC Press, Cleveland, Ohio, 1977. 